\title{
Fluctuating Regional Brainstem Diffusion Imaging Measures of Microstructure across the Migraine Cycle
}

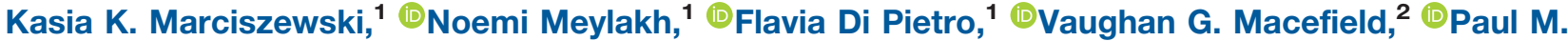 \\ Macey, ${ }^{3}$ and ${ }^{-}$Luke A. Henderson ${ }^{1}$
}

https://doi.org/10.1523/ENEURO.0005-19.2019

\begin{abstract}
${ }^{1}$ Department of Anatomy and Histology, Sydney Medical School, University of Sydney, Sydney, 2006 New South Wales, Australia, ${ }^{2}$ School of Medicine, Western Sydney University, Campbelltown, 2560 New South Wales, Australia, and ${ }^{3}$ University of California, Los Angeles School of Nursing and Brain Research Institute, University of California, Los Angeles, CA 90095
\end{abstract}

\begin{abstract}
The neural mechanisms responsible for the initiation and expression of migraines remain unknown. Although there is growing evidence of changes in brainstem anatomy and function between attacks, very little is known about brainstem function and structure in the period immediately prior to a migraine. The aim of this investigation is to use brainstem-specific analyses of diffusion weighted images to determine whether the brainstem pain processing regions display altered structure in individuals with migraine across the migraine cycle, and in particular immediately prior to a migraine. Diffusion tensor images (29 controls, 36 migraineurs) were used to assess brainstem anatomy in migraineurs compared with controls. We found that during the interictal phase, migraineurs displayed greater mean diffusivity (MD) in the region of the spinal trigeminal nucleus (SpV), dorsomedial pons (dmPons)/dorsolateral pons (dIPons), and midbrain periaqueductal gray matter (PAG)/cuneiform nucleus (CNF). Remarkably, the MD returned to controls levels during the 24-h period immediately prior to a migraine, only to increase again within the three following days. Additionally, fractional anisotropy (FA) was significantly elevated in the region of the medial lemniscus/ventral trigeminal thalamic tract in migraineurs compared with controls over the entire migraine cycle. These data show that regional brainstem anatomy changes over the migraine cycle, with specific anatomical changes occurring in the 24-h period prior to onset. These changes may contribute to the activation of the ascending trigeminal pathway by either an increase in basal traffic or by sensitizing the trigeminal nuclei to external triggers, with activation ultimately resulting in perception of head pain during a migraine attack.
\end{abstract}

Key words: brainstem; diffusion tensor imaging; migraine; MRI; PAG; spinal trigeminal nucleus

\section{Significance Statement}

It has been hypothesized that modulation of brainstem pain pathways may be critical for the initiation of migraine attacks. There is some evidence that altered brainstem function, possibly involving increased astrocyte activation, occurs immediately before a migraine attack. We sought to obtain evidence to support this theory. Using diffusion tensor imaging (DTI), we found that immediately before a migraine, mean diffusivity (MD) decreased in the spinal trigeminal nucleus $(\mathrm{SpV})$, dorsomedial pons (dmPons)/dorsolateral pons (dIPons), and midbrain periaqueductal gray matter (PAG)/nucleus cuneiform. MD then increased again immediately following the migraine attack. Decreased MD before a migraine is consistent with increased astrocyte activation, since astrocyte processes enlarge during activation. These changes may underlie changes in brainstem function that are essential for the generation of a migraine. 


\section{Introduction}

Migraine is a common, debilitating disorder characterized by headaches and often accompanied by aura, nausea, and sensitivity to light and sound. Despite these well-characterized symptoms, the exact mechanisms underlying the initiation and maintenance of migraine head pain are still hotly debated. To date, human brain imaging investigations have revealed that during a migraine attack, activity increases in brain regions such as the cingulate cortex, insula, thalamus, hypothalamus and dorsal pons (Weiller et al., 1995; Bahra et al., 2001; Denuelle et al., 2004; Afridi et al., 2005). In addition, a number of studies have identified anatomical, sensitivity, and resting activity pattern changes between migraine attacks, i.e., in the interictal period (Chong and Schwedt, 2015; Mathur et al., 2016; Chong et al., 2017; Porcaro et al., 2017; Marciszewski et al., 2018a). These findings highlight the apparent brain dysfunction in migraineurs even when in a pain-free state.

A recent review has proposed that these observed changes in brain function are not stable but dynamic in nature (May, 2017). Some have suggested that functional brain changes actually trigger a migraine from basal firing (Goadsby and Akerman, 2012). Others have suggested that the brain fluctuates between a state where the effectiveness of endogenous analgesic mechanisms is too great to allow incoming noxious inputs to evoke head pain, and a state where incoming inputs can activate central pathways and evoke head pain (Akerman et al., 2011; Borsook and Burstein, 2012). Consistent with these hypotheses, it has recently been reported that during the interictal phase, migraineurs display reduced gray matter density and increased free water movement within brainstem pain-modulating regions including the midbrain periaqueductal gray matter (PAG), dorsolateral pons (dIPons), medullary raphe and spinal trigeminal nucleus (SpV; Marciszewski et al., 2018a). Furthermore, it was recently shown that immediately before a migraine resting infraslow oscillatory activity increases in these same brainstem regions and returns to controls levels shortly after the migraine and it was speculated that these oscillatory changes may result from transient increases in astrocyte activation and its associated gliotransmission (Meylakh et al., 2018). Given there is some evidence that astrocytes may play a role in aspects of migraine such as the prop-

Received January 6, 2019; accepted July 8, 2019; First published July 12, 2019

The authors declare no competing financial interests.

Author contributions: K.K.M., N.M., and L.A.H. performed research; K.K.M. and P.M.M. analyzed data; K.K.M., N.M., F.D.P., V.G.M., P.M.M., and L.A.H. wrote the paper; F.D.P. and L.A.H. designed research.

This work was supported by the National Health and Medical Research Council of Australia Grant 1143547

Acknowledgements: We thank the many volunteers in this study.

Correspondence should be addressed to Luke A. Henderson at lukeh@anatomy.usyd.edu.au.

https://doi.org/10.1523/ENEURO.0005-19.2019

Copyright (C) 2019 Marciszewski et al.

This is an open-access article distributed under the terms of the Creative Commons Attribution 4.0 International license, which permits unrestricted use, distribution and reproduction in any medium provided that the original work is properly attributed. agation of cortical spreading depression (Nedergaard et al., 1995) and that a genetic form of migraine, familial hemiplegic migraine, is associated with astrocyte dysfunction (Benarroch, 2005), it is not unreasonable to suggest that astrocytes may also play a critical role in migraine pathophysiology via actions within the brainstem.

It was recently reported that pain sensitivity to noxious stimuli in migraineurs is dramatically decreased in the 24-h period before a migraine, and this decrease is associated with increased functional magnetic resonance imaging (fMRI) signal intensity within the $\mathrm{SpV}$ and reduced PAG-SpV connectivity (Marciszewski et al., 2018b). This altered brainstem function may result from altered neuralglial interactions, although evidence of astrocyte activation in migraineurs, particularly in the period immediately before a migraine is lacking. This is likely due to the fact that it is not possible to predict when an individual will have a migraine and thus examining them in the 24-h period before an attack is exceptionally difficult. Local free water movement, as measured by diffusion tensor imaging (DTI), is a method by which microstructural alterations can be examined in living humans. Such diffusion measures can be altered by numerous processes that change local anatomy, including edema, inflammation, demyelination and alterations in cell numbers and/or morphology. Furthermore, astrocyte activation, which is associated with their processes enlarging considerably, would result in a decrease in local free water movement.

The aim of this investigation is to use DTI to determine whether the brainstem displays microstructural alterations throughout the migraine cycle. More specifically we aim to explore changes in the $24-\mathrm{h}$ period before a migraine. We hypothesize that immediately before a migraine, mean diffusivity (MD) will decrease, consistent with an increase in astrocyte size, in areas of the brainstem that process and modulate pain such the PAG, dIPons, medullary raphe, and $\mathrm{SpV}$. Furthermore, that decrease will be reversed during the period immediately following a migraine and return to interictal levels.

\section{Materials and Methods}

\section{Subjects}

Thirty-six subjects with migraine (eight males, mean \pm SEM age: $30.6 \pm 1.7$ years) and 29 age and gender matched pain-free controls (five males, age: $31.9 \pm 2.3$ years) were recruited for the study. All subjects were recruited from the general population using an advertisement. Migraine subjects were diagnosed according to the criteria laid out by the International Classification of Headache Disorders (ICHD), 3rd edition, sections 1.1 and 1.2 (ICHD-3 $\beta, 2013)$. Seven migraineurs reported aura associated with their migraines and the remaining 29 reported no aura. Of the 36 migraineurs, 31 were scanned during the interictal period (seven males, age $30.1 \pm 1.9$ years), that is, between $72 \mathrm{~h}$ after and $24 \mathrm{~h}$ before a migraine attack; 13 during the 24-h period immediately before a migraine (four males, age $26.0 \pm 2.4$ years), and 15 within the 72-h period following a migraine (four males, age 31.5 \pm 2.5 years). For subjects scanned before an attack, there 
was no predicting factor that they were within $24 \mathrm{~h}$ of a migraine. Eleven migraineurs were scanned during the interictal period and period immediately before a migraine. In addition, seven of these 10 subjects were also scanned during the period immediately after a migraine.

All migraine subjects indicated the pain intensity (sixpoint visual analog scale; $0=$ no pain, $5=$ most intense imaginable pain) and facial distribution (drawing) of pain they commonly experience during a migraine attack. Each subject described the qualities of their migraines and indicated any current treatments used to prevent or abort a migraine once started. Exclusion criteria for controls were the presence of any current pain or chronic pain condition, current use of analgesics, and any neurological disorder. Exclusion criteria for migraineurs were any pain condition other than migraine, and any other neurological disorder. Informed written consent was obtained for all procedures according to the Declaration of Helsinki 7th revision and local Institutional Human Research Ethics Committees approved the study.

\section{MRI acquisition}

Subjects lay supine on the bed of a $3 T$ MRI scanner (Philips Achieva, Neuroscience Research Australia) with their head immobilized in a fitting 32-channel head coil. With all subjects relaxed and at rest, in each subject a high-resolution T1-weighted anatomical image set covering the entire brain was collected (turbo field echo; field of view $250 \times 250 \mathrm{~mm}$, matrix size $=288 \times 288$, slice thickness $=0.87 \mathrm{~mm}$, repetition time $=5600 \mathrm{~ms}$, echo time $=2.5 \mathrm{~ms}$, flip angle $8^{\circ}$ ). Following this, two highresolution DTI image sets covering the entire brain were collected using a single-shot multisection spin-echo echo-planar pulse sequence (repetition time $=8788 \mathrm{~ms}$; flip angle $=90^{\circ}$, matrix size $112 \times 112$, field of view 224 $\times 224 \mathrm{~mm}$, slice thickness $=2.5 \mathrm{~mm}, 55$ axial slices). For each slice, diffusion gradients were applied along 32 independent orientations with $b=1000 \mathrm{~s} / \mathrm{mm}^{2}$ after the acquisition of five $b=0 \mathrm{~s} / \mathrm{mm}^{2}$ (b0) images. Two DTI acquisitions were averaged to improve signal-noise ratios.

\section{Image analysis}

$D T I$ analysis

Using SPM12 software (Friston et al., 1994), the two DTI image sets from each subject were realigned based on the b0 images, and the diffusion tensors calculated from the images using a linear model (Basser and Pierpaoli, 1996). MD, axial diffusivity, radial diffusivity, and fractional anisotropy ( $F A$ ) whole-brain maps were then derived and co-registered to each individual subject's T1-weighted image. Using brainstem-specific isolation software (SUIT toolbox; Diedrichsen, 2006), a unique mask of the brainstem was manually created for each subject's MD map. Using these masks, the brainstem was isolated from the $M D$, axial diffusivity, radial diffusivity and FA maps, spatially normalized, re-sliced to the SUIT template in Montreal Neurologic Institute (MNI) space, and spatially smoothed using a 3-mm full-width-at-half-maximum (FWHM) Gaussian filter.

\section{Statistical analyses}

Using a voxel-by-voxel analysis, significant differences in MD and FA values were determined between (1) controls $(n=29)$ and migraineurs during the interictal period $(n=31)$, (2) controls and migraineurs during the period immediately before a migraine $(n=13)$, and (3) controls and migraineurs during the period immediately following a migraine $(n=15$; all comparisons $p<0.05$, false discovery rate corrected at a voxel level, minimum cluster size 5 contiguous voxels). Age and gender were included as nuisance variables and a brainstem mask that excluded cerebrospinal fluid as well as the cerebellum was applied to each analysis.

Since we found significant MD increases during the interictal period that were eliminated immediately before a migraine, we extracted MD values from those significant clusters for all three migraine periods. Significant MD differences between controls and the period immediately before and following migraine were then determined for these clusters (two-tailed, two-sample $t$ test, $p<0.05$ ). In addition, the axial diffusivity, radial diffusivity and FA values from each of the significant clusters were extracted and plotted and significant differences between controls and each migraine period as well as between migraine periods themselves were determined (two-tailed, twosample $t$ test, $p<0.05$ ). We also found a cluster of significant FA change that was present during all three migraine phases. We extracted the MD, axial diffusivity, radial diffusivity, and FA values from significant clusters of the control versus interictal analysis for all three migraine periods and determined significance relative to controls and between migraine phases (two-tailed, two-sample $t$ test, $p<0.05)$. Significant MD and FA differences between controls and the interictal period were not assessed a second time since these were already established as significant with the voxel-based statistics, thus avoiding the issue of "double-dipping."

To explore changes in individual migraineurs throughout the migraine cycle, in 11 migraineurs that were scanned during more than one period, we plotted MD and FA values for each significant cluster identified in the original group analysis. Significant MD and FA differences between each of the three migraine periods were then determined using paired $t$ tests (two-tailed $p<0.05$ ). Additionally, for all migraineurs, $M D$ and $F A$ values relative to the time until next migraine were plotted and a line of best fit applied for each significant cluster determined in the original group analysis to explore whether MD and FA increased or decreased as a migraine event approached. Finally, for each cluster, significant relationships between $\mathrm{MD}$ and $\mathrm{FA}$ and migraine characteristics were determined (Pearson correlation, $p<0.05$ ).

\section{Results}

\section{Migraine characteristics}

Using a self-report questionnaire, migraineurs reported the most common location of their migraines over the past 12 months. In 12 of the 36 migraineurs, headaches were more common on the right side, in six they were more common on the left, and in the remaining 18 they were 
most often bilateral. Migraine subjects most frequently described their migraine pain as "throbbing," "pulsating," and/or "sharp" in nature. They indicated that "stress," "lack of sleep," and "dehydration" most often triggered migraine attacks. The mean ( \pm SEM) estimated frequency of migraine attacks was $1.3 \pm 0.1$ per month, mean length of time since the onset of migraine attacks (years suffering) $16.2 \pm 1.9$ years, and mean pain intensity of migraines was $3.8 \pm 0.1$ on a six-point visual analog scale. Although 24 of the 36 migraineurs were taking some form of daily medication (mostly the oral contraceptive pill), none of the migraine subjects were taking prophylactic medication prescribed for migraine.

\section{Diffusivity measures}

\section{Group comparisons}

The DTI analysis revealed that compared to controls, migraineurs show regional differences in brainstem MD throughout the migraine cycle (Fig. 1; Table 1). Consistent with our previous report, during the interictal period migraineurs showed increased MD compared with controls in regions encompassing the left $\mathrm{SpV}$, left dIPons, right dorsomedial pons (dmPons)/dIPons, and the region of the midbrain PAG and including the region of the cuneiform nucleus (CNF; Fig. 1; Table 1). Strikingly, this MD increase during the interictal period was absent during the 24-h period before a migraine, with no significant difference between controls and migraineurs in this period. The MD increase then returned to above control levels in the $\mathrm{dm} / \mathrm{dlPons}$ and PAG/CNF in the 72-h period immediately following a migraine. Extraction of $M D$ values from the clusters displaying a significant increase during the interictal period confirmed this pattern of MD change, i.e., MD increases during the interictal, no MD difference immediately before migraine and MD increase again immediately following a migraine (Fig. 2; Table 2). In no brainstem region was $\mathrm{MD}$ significantly lower in migraineurs compared with controls.

Extraction of axial and radial diffusion values from these brainstem regions resulted in an interesting pattern of change (Table 2). Within the SpV, as with MD, both axial and radial diffusivity was significantly increased only during the interictal phase, and within the PAG/CNF axial and radial diffusivity was significantly increased during the interictal phase but unlike MD, it was not increased during the phase immediately following a migraine. Interestingly, within the left and right dIPons and dmPons regions, while axial diffusivity was significantly increased during the interictal and immediately following migraine phases, radial diffusivity was not significantly different during any migraine phase compared with controls and FA was not different during either phase or compared with controls.

Analysis of FA revealed a different pattern of change to that of the other diffusion measures. Migraineurs displayed a significant increase in FA in the area encompassing the ventral trigeminothalamic tract/ventral tegmental area (VTT/NTA) during all three phases (Fig. 1). Extraction of $F A$ values from this region confirmed that FA was significantly increased in migraineurs and that this in- crease was apparent during all migraine phases (Fig. 2; Table 2).

\section{Individual migraineur comparisons}

Plots of MD values in the 11 migraineurs that were scanned during at least two of the three migraine periods revealed that the pattern of MD changes was consistent in individual subjects. That is, MD was lower immediately before a migraine compared with both the interictal and immediately following a migraine period (Fig. 3). While the left dIPons did not show a significant difference between phases, paired $t$ tests revealed that the left $\mathrm{SpV}$, right dIPons and left PAG/CNF displayed significantly reduced MD during the phase immediately before a migraine compared with both the interictal and immediately following a migraine phase. More specifically, of the 11 migraineurs, MD decreased immediately before a migraine compared to the interictal period in 10 migraineurs within the left $\mathrm{SpV}$, left PAG/CNF, and right $\mathrm{dm} / \mathrm{dIP}$ ons and in nine migraineurs in the left dIPons. Additionally, of the seven migraineurs scanned during all three phases, all showed a MD decrease immediately before a migraine compared with both the interictal and immediately after a migraine within the left SpV, and five migraineurs for the left dIPons, right dm/dIPons, and PAG/CNF. In contrast to MD, consistent with the group analysis, FA was relatively consistent between the migraine phases, although it was significantly increased during the immediately before compared with the immediately following migraine phase.

Furthermore, plots of MD and FA values over the migraine cycle revealed that within the SpV, right and left dIPons, and PAG/CNF, MD appeared to remain relatively stable across the interictal period before decreasing immediately before the migraine and then increasing again after the migraine (Fig. 4). In contrast, the elevated FA in the VTT/NTA remained relatively stable across the migraine cycle and did not change immediately before a migraine. Finally, in all migraine subject groups, MD or FA values in these clusters during the interictal phase were not significantly correlated to migraine frequency (MD: left SpV $r=0.03$, left dIPons $r=-0.13$, right dm/dIPons $r=$ 0.15 , left PAG/CNF: $r=0.01$; FA: VTTNTA: $r=0.09$; all $p$ $>0.05$ ), years suffering (MD: left SpV $r=0.11$, left dIPons $r=-0.09$, right dm/dIPons $r=-0.30$, left PAG/CNF $r=$ 0.14 ; FA: VTT/VTA $r=0.14$; all $p>0.05)$, or the intensity of migraine pain (left SpV $r=0.31$, left dlPons $r=-0.11$, right dm/dIPons $r=-0.03$, left PAG/CNF $r=0.09$; FA: VTTNTA $r=-0.03$; all $p>0.05$ ).

\section{Discussion}

This study demonstrates that migraine is associated with changes in regional anatomy that fluctuate over the migraine cycle in a number of brainstem regions. More specifically, during the long interictal period, migraineurs display increased free water movement compared with controls in areas that process orofacial pain, such as the SpV, dIPons, and PAG/CNF. Remarkably, immediately before a migraine attack, this increase in diffusivity reduces to control levels before increasing again in the period immediately following migraine. It is clear from these data that in episodic migraineurs, regional brains- 


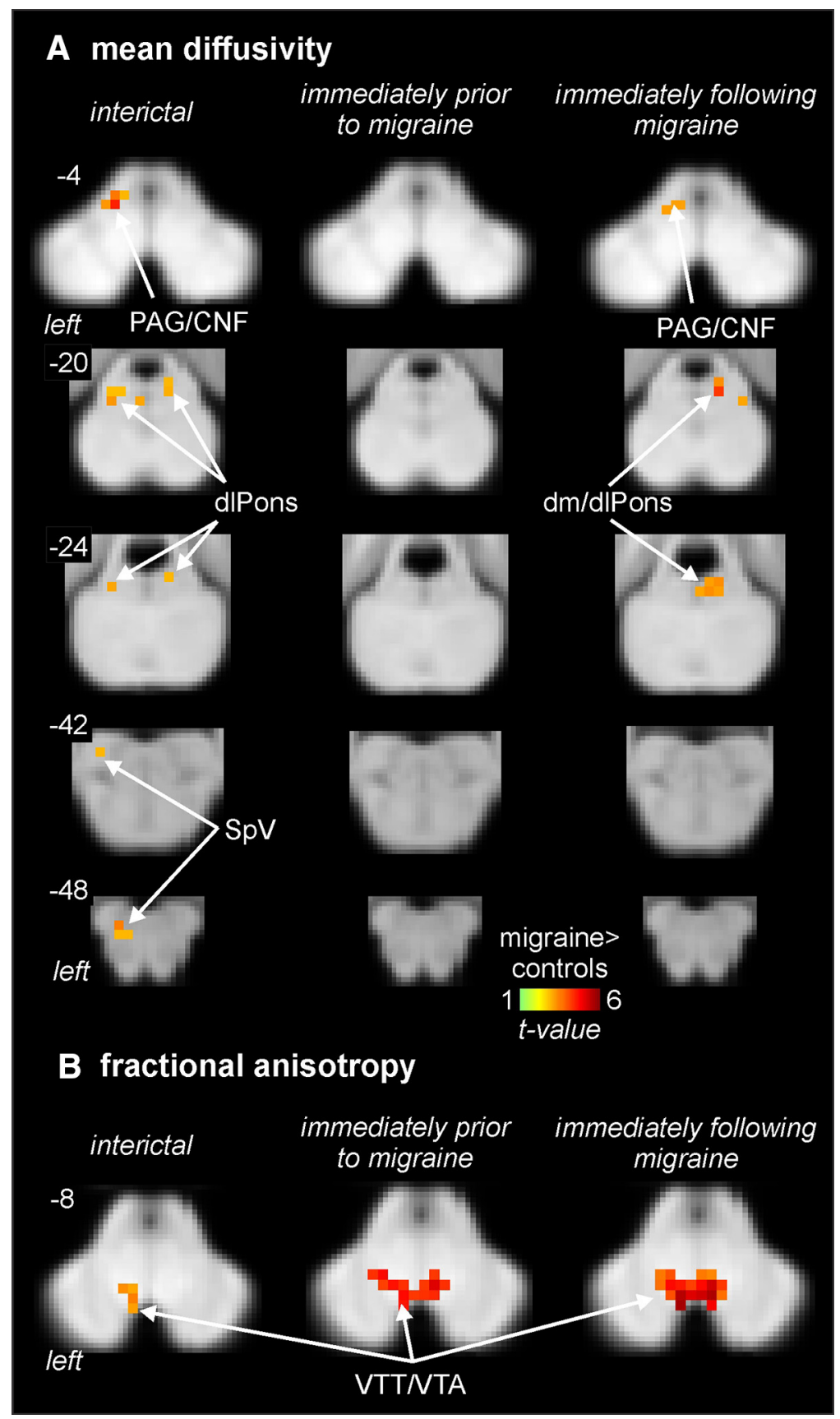

Figure 1. Regional MD $(\boldsymbol{A})$ and FA differences $(\boldsymbol{B})$ in migraineurs during the interictal $(n=31)$, immediately before migraine $(n=13)$, and immediately following a migraine $(n=15)$ compared with controls $(n=29)$. Significantly different clusters are overlaid onto axial brainstem template images and significant increases in migraineurs are represented by a $t$ value with a hot color scale. Slice locations are indicated at the upper left of each axial slice in MNI space. Compared to controls, migraineurs have increased MD during the interictal and immediately following migraine periods in the region of the left $\mathrm{SpV}$, left and right dIPons, and in the region encompassing the PAG/CNF. In addition, migraineurs display an increase in FA in the region of the VTT/VTA during all three phases compared with controls.

tem microstructural changes occur throughout the migraine cycle, and that there are specific anatomical changes in the $24 \mathrm{~h}$ before onset.

A number of migraine studies have used DTI to identify anatomical changes in large fiber bundles such as the corpus callosum, internal capsule and corona radiata, although these studies did not explore changes in diffusion within gray matter or within fiber bundles in the brainstem (Neeb et al., 2015; Zhang et al., 2017). While a previous investigation used a region of interest approach to find that migraineurs show greater MD compared to controls within the red nucleus (Kara et al., 2013), no study has specifically explored the brainstem, particularly at different times over the migraine cycle. Consistent with a previous study, we found significant MD increases in SpV, dIPons and PAG/CNF (Marciszewski et al., 2018a), 
Table 1. MNI coordinates, cluster size, and $t$ score for regions of significant increases in MD and FA in migraineurs compared with controls

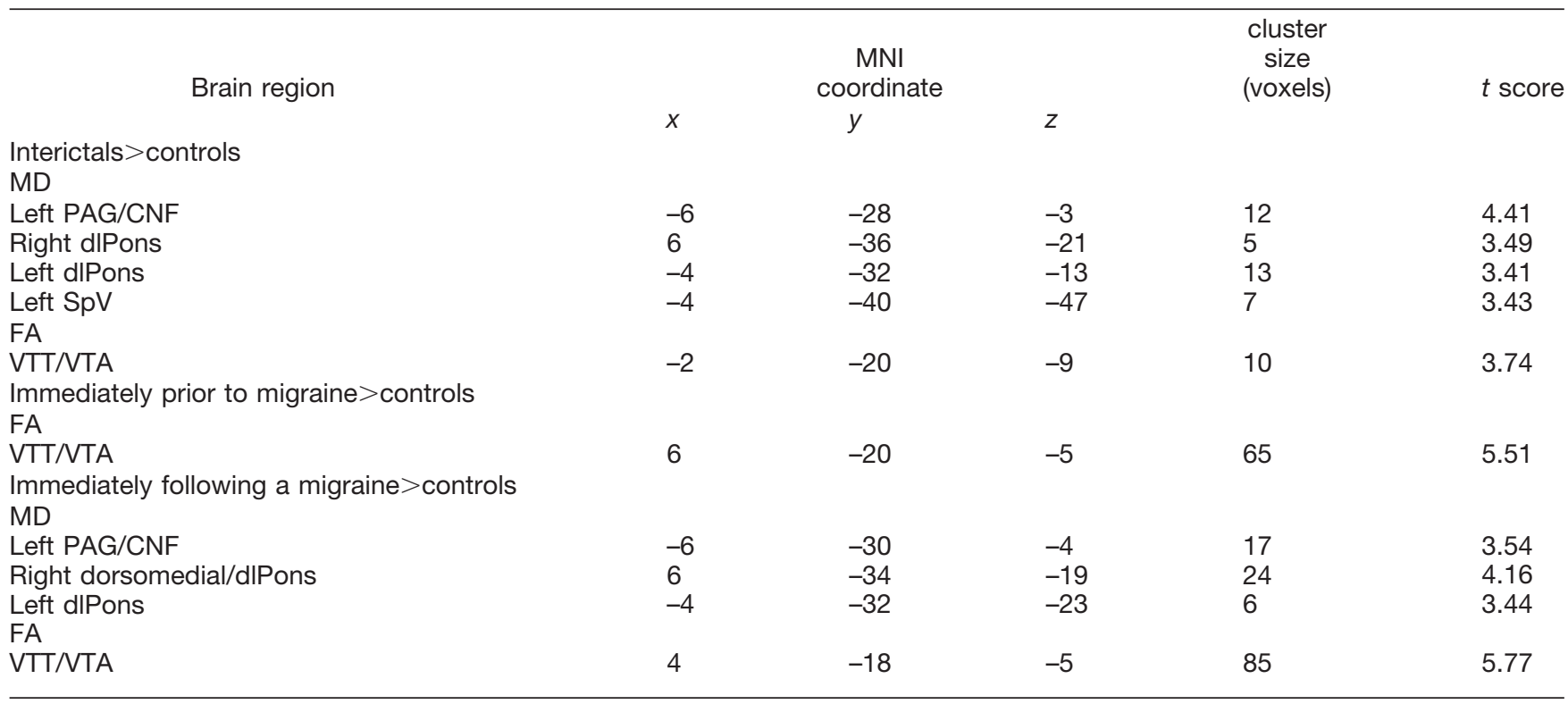

and furthermore, we show that during the 24-h period immediately before a migraine, these structural changes disappear so that migraineurs are no different from controls in the preictal period. However, these structural changes then return during the 72-h period following the migraine event. Furthermore, we found increased FA in the area encompassing the ascending VTT/NTA in migraineurs during all phases.

It has been argued by many that the headache period of migraine results from activation of trigeminal afferents in brain meninges and large cerebral arteries and these afferents terminate in the SpV and upper cervical dorsal horn (Liu et al., 2004, 2008; Olesen et al., 2009). While the nature of the cellular changes underlying such diffusion changes is unclear, MD changes can be associated with edema, vascular injury, inflammation, demyelination, cell count, and cellular morphology; as such our findings could therefore reflect several underlying biological changes (Alexander et al., 2007; Hutchinson et al., 2018). The dynamic nature of the changes reported here suggest that they reflect processes that are not permanent or static in nature, but that can instead change relatively rapidly, at least over the period of a day. Since MD changes can result from dynamic processes such as gliosis (Sierra et al., 2011), and there is evidence that migraine is associated with altered glial function (Nedergaard et al., 1995; Benarroch, 2005), it is possible that the MD decreases immediately before a migraine result from astrocyte activation and associated expansion of astrocytic processes. Indeed, a recent preclinical epilepsy investigation linked microstructural changes in astrocytic processes with altered measures of diffusivity (Salo et al., 2017).

Consistent with the idea that migraine is associated with astrocyte activation, it was recently shown that immediately before a migraine, resting infra-slow oscillatory activity $(0.03-0.06 \mathrm{~Hz})$ increases in these same brainstem regions (Meylakh et al., 2018). Astrocytes can exhibit infra-slow calcium oscillations that can propagate among surrounding astrocytes and it has been proposed that in pathologic situations, enhanced calcium-wave synchrony and amplitude may occur which can alter local neural function (Parri and Crunelli, 2001; Crunelli et al., 2002; Cunningham et al., 2006; Halassa et al., 2007; Lörincz et al., 2009). This raises the prospect that immediately before a migraine, astrocyte activation results in decreased MD and increased infra-slow oscillatory activity resulting in altered sensitivity within brainstem regions that receive and process orofacial noxious information. Whether such a sensitivity change is adequate to evoke head pain from basal levels of neural traffic or simply to facilitate an incoming trigger to activate higher brain centers to produce head pain remains to be determined.

The hypothesis that the brainstem pain processing sites become more sensitive as a migraine approaches was supported by Stankewitz and colleagues and Lee and colleagues, who reported that orofacial noxious and nonnoxious stimuli evoked greater $\mathrm{SpV}$ signal intensity increases as a migraine attack approaches, although no significant change in the perceived intensity of orofacial stimuli (Stankewitz et al., 2011; Lee et al., 2017). Similarly, a recent study by Marciszewski and colleagues also reported that noxious stimuli evoked dramatic SpV activation increases, particularly in the 24-h period before a migraine (Marciszewski et al., 2018b). However, despite the increase in $\mathrm{SpV}$ activation, during acute orofacial stimuli, individuals' reported pain intensity ratings decreased as a migraine approached. This appears at odds with the idea that brainstem pain-processing circuits become more sensitive as a migraine approaches although there are a number of potential explanations: (1) since these data reveal that both the anatomy and function of brainstem pain processing circuits are dynamic, it is possible that these pathways may change again at the onset 

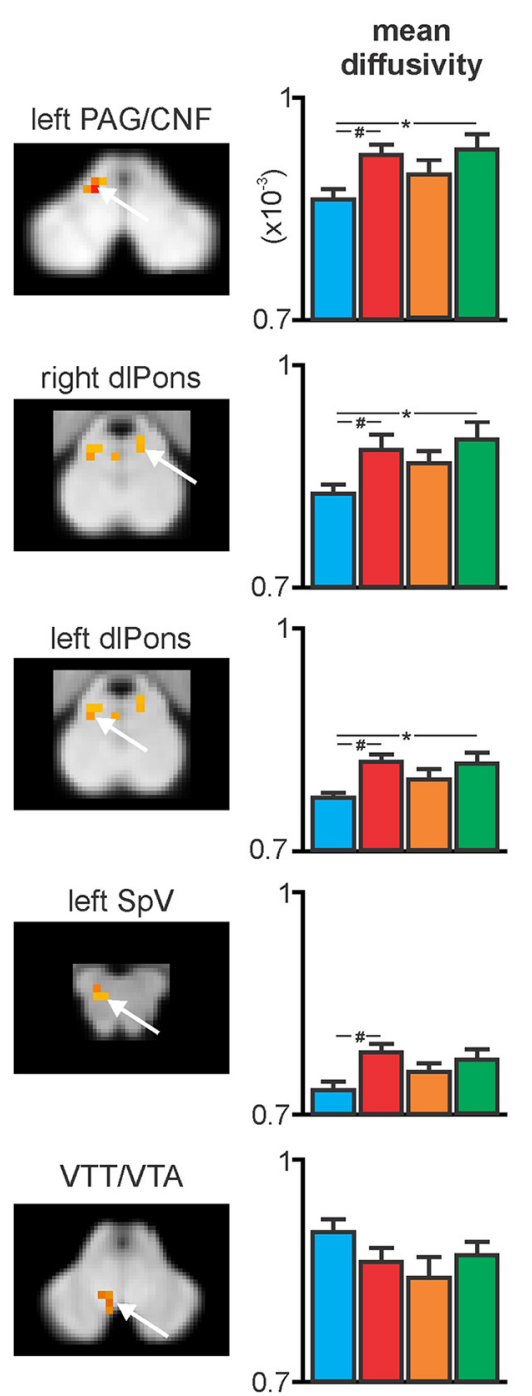
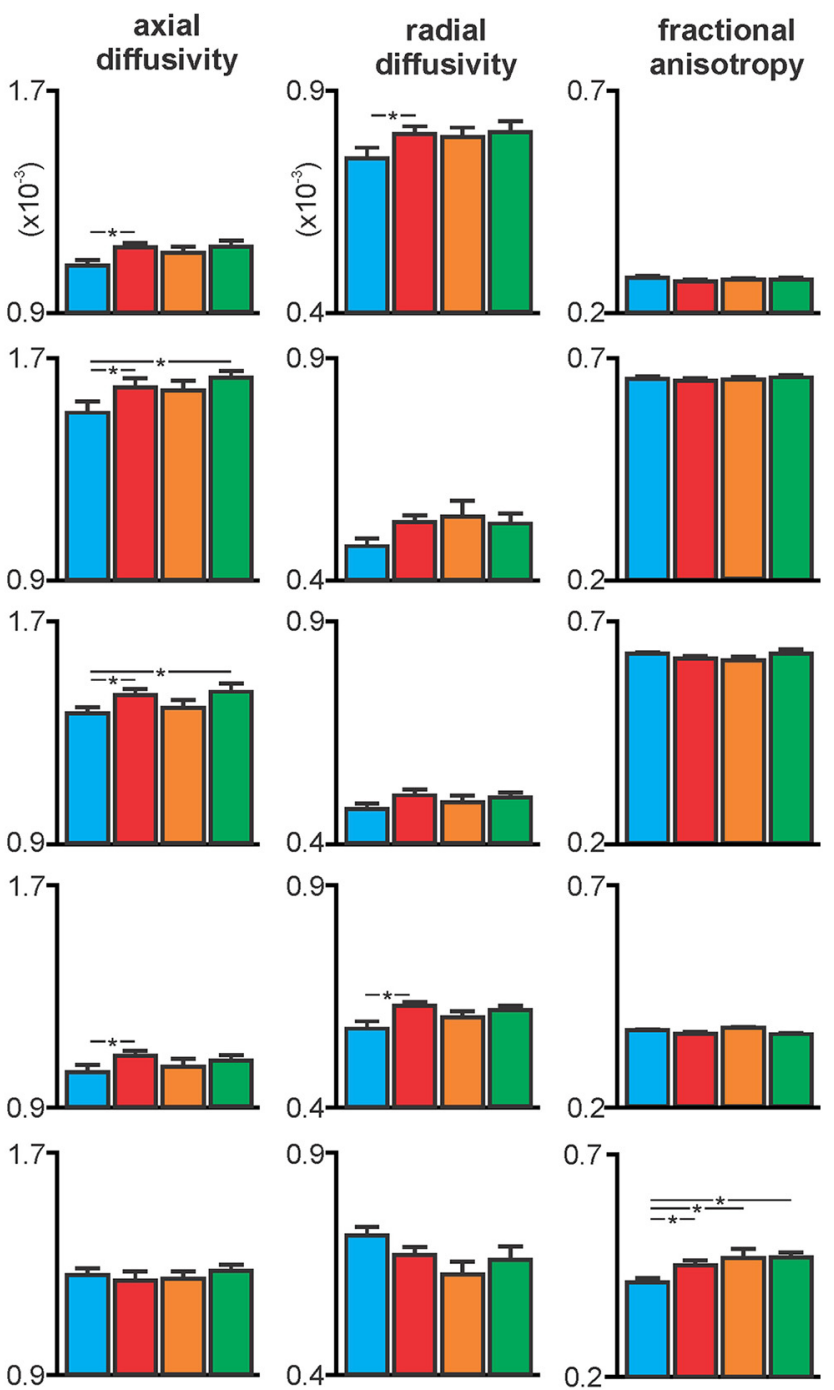

controls $(n=29)$

interictal $(n=31)$

immediately prior to a migraine $(n=13)$

immediately following a migraine $(n=15)$

Figure 2. Plots of mean ( \pm SEM) MD, axial diffusivity, radial diffusivity, and FA values in migraineurs compared with pain-free controls in the left SpV, left and right dIPons, left PAG/CNF, and in the VTT/VTA. Consistent with the voxel-by-voxel analysis, MD was significantly increased during the interictal and immediately following a migraine phase but not immediately before a migraine. In addition, axial diffusivity showed a largely similar pattern of difference in migraineurs whereas radial diffusion was only different during the interictal phase in the PAG/CNF and SpV. In contrast, FA was significantly increased in the area of the VTT/VTA in migraineurs during all three phases; \#p 0.05 voxel-by-voxel analysis; $* p<0.05$ two-sample $t$ tests.

or during a migraine itself; (2) since preclinical studies have shown convergence of dural-sensitive and facial cutaneous afferents in SpV (Burstein et al., 1998; Ellrich et al., 1999), a decrease in noxious cutaneous afferent drive onto second-order convergent SpV neurons may result in an overall increase in dural afferent input sensitivity; (3) changes in descending brainstem modulatory inputs onto the SpV may evoke a heightening of dural afferent input sensitivity at the expense of inputs from other orofacial structures. While these ideas are highly speculative, it is unlikely that the alterations in SpV anatomy and function are involved in other functions to the same degree as the processing of orofacial noxious afferents.
While our data imply that the processes involved in migraine attack onset may be astrocytic in nature, whether astrocyte activity is specifically driving migraine initiation or simply a symptom of another process cannot currently be discerned. The gradual increase in MD in brainstem pain-related regions over the interictal period suggests that changes are occurring throughout the interictal period that then dramatically reverse immediately before a migraine to control levels. While brainstem functional measures such as SpV signal intensity changes and infra-slow oscillations were at control levels during the interictal period and dramatically increased immediately before a migraine, MD was above control levels during the interictal period and reduced to control levels before mi- 
Table 2. Diffusion values for clusters displaying significant increases in MD or FA during the interictal phase in migraineurs compared with controls

\begin{tabular}{llcccc}
\hline $\begin{array}{c}\text { Brain } \\
\text { region }\end{array}$ & Diffusivity parameter & Controls & Interictal & Immediately prior to migraine & Immediately following migraine \\
Left PAG/CNF & MD & $0.86 \pm 0.01$ & $0.92 \pm 0.01$ & $0.89 \pm 0.01$ & $0.93 \pm 0.02$ \\
& Axial diffusivity & $1.07 \pm 0.02$ & $1.14 \pm 0.01$ & $1.12 \pm 0.02$ & $1.14 \pm 0.02$ \\
& Radial diffusivity & $0.75 \pm 0.01$ & $0.81 \pm 0.01$ & $0.79 \pm 0.02$ & $0.81 \pm 0.02$ \\
& FA & $0.28 \pm 0.01$ & $0.27 \pm 0.01$ & $0.27 \pm 0.01$ & $0.27 \pm 0.01$ \\
left dIPons & MD & $0.77 \pm 0.01$ & $0.82 \pm 0.01$ & $0.79 \pm 0.01$ & $0.82 \pm 0.01$ \\
& Axial diffusivity & $1.37 \pm 0.02$ & $1.44 \pm 0.01$ & $1.40 \pm 0.02$ & $1.45 \pm 0.02$ \\
& Radial diffusivity & $0.47 \pm 0.01$ & $0.51 \pm 0.01$ & $0.49 \pm 0.01$ & $0.50 \pm 0.02$ \\
right dI/dmPons & MD & $0.63 \pm 0.01$ & $0.61 \pm 0.01$ & $0.61 \pm 0.01$ & $0.63 \pm 0.01$ \\
& FA & $0.83 \pm 0.01$ & $0.89 \pm 0.1$ & $0.86 \pm 0.01$ & $0.90 \pm 0.01$ \\
& Axial diffusivity & $1.52 \pm 0.02$ & $1.61 \pm 0.02$ & $1.58 \pm 0.02$ & $1.64 \pm 0.02$ \\
SpV & Radial diffusivity & $0.49 \pm 0.01$ & $0.54 \pm 0.02$ & $0.55 \pm 0.03$ & $0.53 \pm 0.02$ \\
& FA & $0.66 \pm 0.01$ & $0.65 \pm 0.01$ & $0.65 \pm 0.02$ & $0.66 \pm 0.01$ \\
& MD & $0.73 \pm 0.01$ & $0.79 \pm 0.01$ & $0.76 \pm 0.1$ & $0.78 \pm 0.1$ \\
& Axial diffusivity & $1.04 \pm 0.02$ & $1.10 \pm 0.01$ & $1.06 \pm 0.01$ & $1.08 \pm 0.01$ \\
VTT/VTA & Radial diffusivity & $0.59 \pm 0.01$ & $0.63 \pm 0.01$ & $0.61 \pm 0.01$ & $0.62 \pm 0.01$ \\
& FA & $0.37 \pm 0.01$ & $0.36 \pm 0.01$ & $0.37 \pm 0.01$ & $0.36 \pm 0.01$ \\
& MD & $0.90 \pm 0.02$ & $0.87 \pm 0.02$ & $0.84 \pm 0.02$ & $0.87 \pm 0.01$ \\
& Axial diffusivity & $1.26 \pm 0.02$ & $1.25 \pm 0.02$ & $1.25 \pm 0.02$ & $1.29 \pm 0.02$ \\
& Radial diffusivity & $0.72 \pm 0.02$ & $0.67 \pm 0.02$ & $0.64 \pm 0.03$ & $0.67 \pm 0.01$ \\
& FA & $0.41 \pm 0.01$ & $0.45 \pm 0.01$ & $0.47 \pm 0.02$ & $0.47 \pm 0.01$
\end{tabular}

MD, axial diffusivity, and radial diffusivity values are expressed as $\times 10^{-3}$. The gray shaded boxes indicate a significant difference compared with controls.

graine. This implies that brainstem anatomy is not simply changing before a migraine but is altered throughout the long interictal period.

Several reports suggest reduced endogenous analgesic ability in migraine (Sandrini et al., 2006; de Tommaso et al., 2007) while others report no change (Perrotta et al., 2010; Teepker et al., 2014). This inconsistency may reflect subtle variations in endogenous analgesic responsiveness across the migraine cycle, and it might be that endogenous analgesic ability gradually increases over the interictal period which is consistent with MD increases in pain-processing and modulating regions across the interictal period. Additionally, none of the regional microstructural changes we detected were correlated to migraine properties such as migraine frequency, intensity or duration, suggesting that the changes are not cumulative over time, and is consistent with the idea that they may be dynamic in nature. While these results are in line with some migraine studies (Chen et al., 2016; Uggetti et al., 2017), others have reported significant linear relationships between anatomical measures and migraine frequency (Kruit et al., 2009; Mainero et al., 2011), intensity (Russo
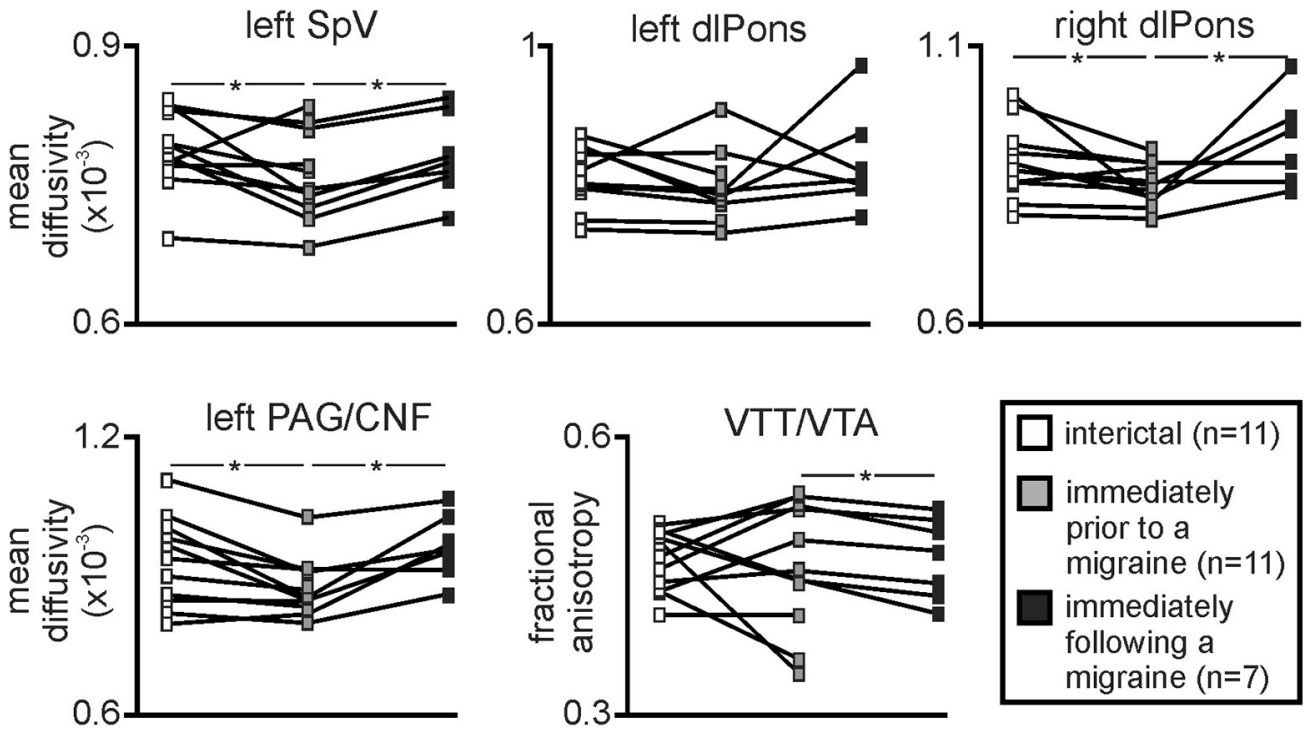

Figure 3. Plots of MD and FA in 11 migraineurs that were scanned during at least two of the three migraine phases. Note the consistency of MD change which decreases significantly during the period immediately before migraine in the left SpV, right dIPons, and left PAG/CNF. In contrast, FA within the area of the VTT/NTA was significantly greater during the period immediately before compared with immediately following a migraine; $* p<0.05$ paired $t$ tests. 

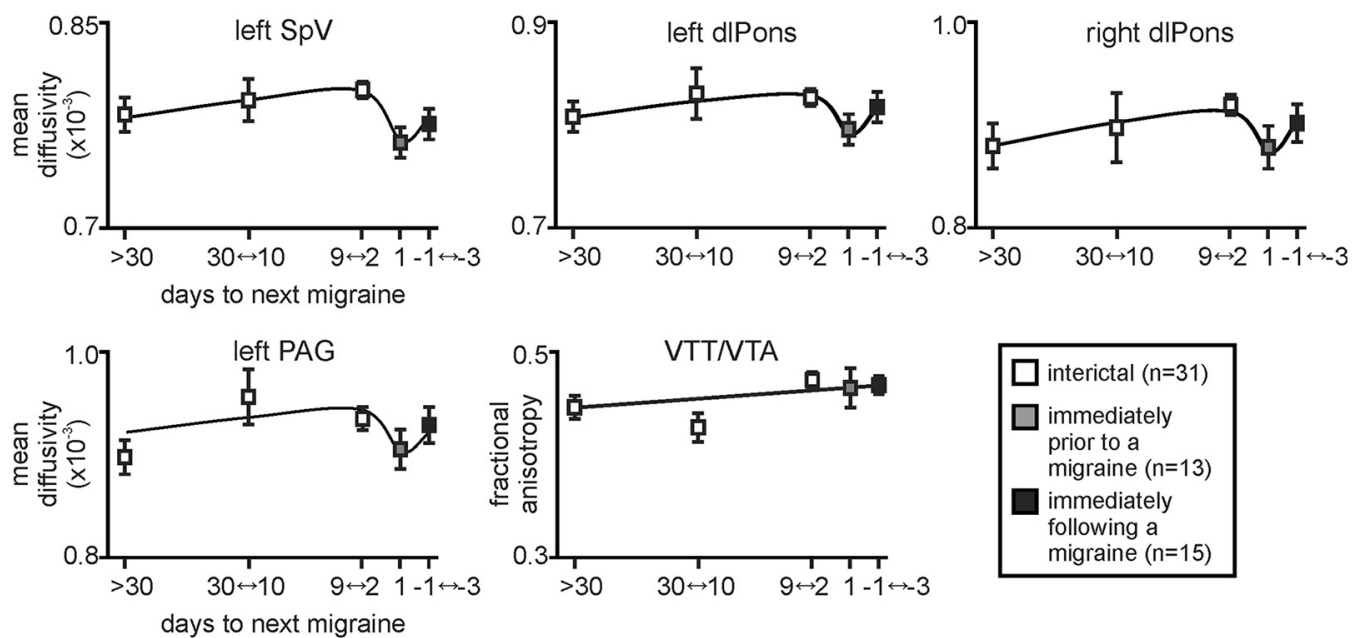

Figure 4. Plots of mean $( \pm S E M) M D$ and FA changes in migraineurs over the migraine cycle in the left SpV, left and right dlPons, left PAG/CNF, and in the VTT/VTA. Values are averaged for those migraineurs scanned at least $30 \mathrm{~d}(n=18), 30-10 \mathrm{~d}(n=6), 9-2 \mathrm{~d}(n$ $=7)$, and $1 \mathrm{~d}$ before their next migraine $(n=13)$, as well as $1-3 \mathrm{~d}$ following a migraine $(n=15)$. Note how MD remains relatively stable over the interictal period and falls dramatically immediately before a migraine before recovering. In contrast, FA remains stable over all three migraine phases.

et al., 2012), and years suffering (Liu et al., 2012; Schwedt et al., 2013; Rocca et al., 2014; Chong and Schwedt, 2015); however, none of these studies explored the brainstem.

In addition to diffusion changes associated with brainstem nuclei, we found FA increases in migraneurs that encompassed the VTT/NTA. These FA increases were not dynamic and occurred at all migraine phases, indicating a constant underlying change, possibly in the ascending trigeminal pain pathway from SpV to the thalamus. While it has been suggested that altered radial diffusion within a fiber tract may represent increases in response to demyelination (Song et al., 2005) and axial diffusivity to axonal damage (Song et al., 2003; Loy et al., 2007), we found no change in axial or radial diffusivity associated with the increase in FA; although it is unclear how accurately the directional diffusivities relate to specific pathologies. Furthermore, while we found primarily changes in axial and not radial diffusivity in brainstem nuclei, what these changes represent remains unknown, although it is not inconsistent with altered astrocyte activation.

There are a number of methodological and subjectrelated limitations of this study. The spatial resolution of human DTI is relatively low and thus it is difficult to precisely localize each brainstem cluster with respect to small brainstem nuclei. However, the location of each cluster was defined using brainstem atlases and we placed the changes into context with respect to the existing human and preclinical research. Secondly, although we managed to scan 13 migraineurs immediately before a migraine, only seven migraineurs were scanned during all three phases and we did not scan migraineurs during a migraine attack itself. However, we did use populationbased statistics with thresholds corrected for multiple comparisons, and although a larger subject number is always preferable, we are confident our results are robust. Future studies in which the migraine phase itself was also explored would further increase our understanding of an- atomical changes across the migraine cycle. Thirdly, this is a largely cross-sectional study and with mounting evidence of relatively rapid brainstem changes, scanning individual migraineurs over the course of several weeks while measuring indices of brain anatomy, activity, and sensitivity would provide more precise evidence supporting this hypothesis. Furthermore, although the diffusivity changes reported here are dynamic in nature, i.e., over a period of days, it remains unknown if such changes are dynamic enough to be altered by transient activation of cell populations.

Overall, our findings suggest that migraine is associated with anatomical changes within brainstem structures involved in trigeminal noxious transmission and endogenous analgesia. More importantly, these anatomical changes alter over the migraine cycle specifically during the 24-h period before a migraine attack. We speculate that these anatomical changes reflect astrocyte activation that alters local neural function by the release of gliotransmitters, which either trigger or alter the sensitivity of the brainstem so that an external trigger induces a migraine attack. Future investigations exploring brainstem resting activity, evoked activity and anatomy over the migraine cycle may provide evidence supporting such a proposal. If dynamic changes in brainstem function and structure do occur, we may be in a position to modify these changes and potentially prevent the triggering of a migraine attack.

\section{References}

Afridi SK, Giffin NJ, Kaube H, Friston KJ, Ward NS, Frackowiak RS, Goadsby PJ (2005) A positron emission tomographic study in spontaneous migraine. Arch Neurol 62:1270-1275.

Akerman S, Holland PR, Goadsby PJ (2011) Diencephalic and brainstem mechanisms in migraine. Nat Rev Neurosci 12:570-584.

Alexander AL, Lee JE, Lazar M, Field AS (2007) Diffusion tensor imaging of the brain. Neurotherapeutics 4:316-329. 
Bahra A, Matharu MS, Buchel C, Frackowiak RS, Goadsby PJ (2001) Brainstem activation specific to migraine headache. Lancet 357: 1016-1017.

Basser PJ, Pierpaoli C (1996) Microstructural and physiological features of tissues elucidated by quantitative-diffusion-tensor MRI. J Magn Reson B 111:209-219.

Benarroch EE (2005) Neuron-astrocyte interactions: partnership for normal function and disease in the central nervous system. Mayo Clin Proc 80:1326-1338.

Borsook D, Burstein R (2012) The enigma of the dorsolateral pons as a migraine generator. Cephalalgia 32:803-812.

Burstein R, Yamamura H, Malick A, Strassman AM (1998) Chemical stimulation of the intracranial dura induces enhanced responses to facial stimulation in brain stem trigeminal neurons. J Neurophysiol 79:964-982.

Chen Z, Chen X, Liu M, Liu S, Ma L, Yu S (2016) Nonspecific periaqueductal gray lesions on T2WI in episodic migraine. J Headache Pain 17:101.

Chong CD, Plasencia JD, Frakes DH, Schwedt TJ (2017) Structural alterations of the brainstem in migraine. Neuroimage Clin 13:223227.

Chong CD, Schwedt TJ (2015) Migraine affects white-matter tract integrity: a diffusion-tensor imaging study. Cephalalgia 35:11621171.

Crunelli V, Blethyn KL, Cope DW, Hughes SW, Parri HR, Turner JP, Tòth TI, Williams SR (2002) Novel neuronal and astrocytic mechanisms in thalamocortical loop dynamics. Philos Trans R Soc Lond B Biol Sci 357:1675-1693.

Cunningham MO, Pervouchine DD, Racca C, Kopell NJ, Davies CH, Jones RS, Traub RD, Whittington MA (2006) Neuronal metabolism governs cortical network response state. Proc Natl Acad Sci USA 103:5597-5601.

Denuelle M, Fabre N, Payoux P, Chollet F, Geraud G (2004) Brainstem and hypothalamic activation in spontaneous migraine attacks. Cephalalgia 24:775-814.

de Tommaso M, Difruscolo O, Sardaro M, Libro G, Pecoraro C, Serpino C, Lamberti P, Livrea P (2007) Effects of remote cutaneous pain on trigeminal laser-evoked potentials in migraine patients. $J$ Headache Pain 8:167-174.

Diedrichsen $J$ (2006) A spatially unbiased atlas template of the human cerebellum. Neurolmage 33:127-138.

Ellrich J, Andersen OK, Messlinger K, Arendt-Nielsen L (1999) Convergence of meningeal and facial afferents onto trigeminal brainstem neurons: an electrophysiological study in rat and man. Pain 82:229-237.

Friston KJ, Holmes AP, Worsley KJ, Poline JP, Frith CD, Frackowiak RS (1994) Statistical parametric maps in functional imaging: a general linear approach. Hum Brain Mapp 2:189-210.

Goadsby PJ, Akerman S (2012) The trigeminovascular system does not require a peripheral sensory input to be activated-migraine is a central disorder. Focus on 'Effect of cortical spreading depression on basal and evoked traffic in the trigeminovascular sensory system.' Cephalalgia 32:3-5.

Halassa MM, Fellin T, Haydon PG (2007) The tripartite synapse: roles for gliotransmission in health and disease. Trends Mol Med 13:5463.

Hutchinson EB, Schwerin SC, Avram AV, Juliano SL, Pierpaoli C (2018) Diffusion MRI and the detection of alterations following traumatic brain injury. J Neurosci Res 96:612-625.

ICHD-3 $\beta$ (2013) International classification committee of the international headache society: international classification for headache disorders (ICHD)-3 $\beta$ manual. Cephalalgia 33:629-808.

Kara B, Kiyat Atamer A, Onat L, Ulusoy L, Mutlu A, Sirvanci M (2013) DTI findings during spontaneous migraine attacks. Clin Neuroradiol 23:31-36.

Kruit MC, Launer LJ, Overbosch J, van Buchem MA, Ferrari MD (2009) Iron accumulation in deep brain nuclei in migraine: a population-based magnetic resonance imaging study. Cephalalgia 29:351-359.
Lee J, Lin RL, Garcia RG, Kim J, Kim H, Loggia ML, Mawla I, Wasan AD, Edwards RR, Rosen BR, Hadjikhani N, Napadow V (2017) Reduced insula habituation associated with amplification of trigeminal brainstem input in migraine. Cephalalgia 37:1026-1038.

Liu J, Zhao L, Li G, Xiong S, Nan J, Li J, Yuan K, Von Deneen KM, Liang F, Qin W, Tian J (2012) Hierarchical alteration of brain structural and functional networks in female migraine sufferers. PLoS One 7:e51250.

Liu Y, Broman J, Edvinsson L (2004) Central projections of sensory innervation of the rat superior sagittal sinus. Neuroscience 129: 431-437.

Liu Y, Broman J, Edvinsson L (2008) Central projections of the sensory innervation of the rat middle meningeal artery. Brain Res 1208:103-110.

Lörincz ML, Geall F, Bao Y, Crunelli V, Hughes SW (2009) ATPdependent infra-slow $(<0.1 \mathrm{~Hz})$ oscillations in thalamic networks. PLoS One 4:e4447.

Loy DN, Kim JH, Xie M, Schmidt RE, Trinkaus K, Song SK (2007) Diffusion tensor imaging predicts hyperacute spinal cord injury severity. J Neurotrauma 24:979-990.

Mainero C, Boshyan J, Hadjikhani N (2011) Altered functional magnetic resonance imaging resting-state connectivity in periaqueductal gray networks in migraine. Ann Neurol 70:838-845.

Marciszewski KK, Meylakh N, Di Pietro F, Macefield VG, Macey PM, Henderson LA (2018a) Altered brainstem anatomy in migraine. Cephalalgia 38:476-486.

Marciszewski KK, Meylakh N, Di Pietro F, Mills EP, Macefield VG, Macey PM, Henderson LA (2018b) Changes in brainstem pain modulation circuitry function over the migraine cycle. J Neurosci 38:10479-10488.

Mathur VA, Moayedi M, Keaser ML, Khan SA, Hubbard CS, Goyal M, Seminowicz DA (2016) High frequency migraine is associated with lower acute pain sensitivity and abnormal insula activity related to migraine pain intensity, attack frequency, and pain catastrophising. Front Hum Neuroci 10:489.

May A (2017) Understanding migraine as a cycling brain syndrome: reviewing the evidence from functional imaging. Neurol Sci 38 [Suppl 1]:125-130.

Meylakh N, Marciszewski KK, Di Pietro F, Macefield VG, Macey PM, Henderson LA (2018) Deep in the brain: changes in subcortical function immediately preceding a migraine attack. Hum Brain Mapp 39:2651-2663.

Nedergaard M, Cooper AJ, Goldman SA (1995) Gap junctions are required for the propagation of spreading depression. J Neurobiol 28:433-444.

Neeb L, Bastian K, Villringer K, Gits HC, Israel H, Reuter U, Fiebach JB (2015) No microstructural white matter alterations in chronic and episodic migraineurs: a case-control diffusion tensor magnetic resonance imaging study. Headache 55:241-251.

Olesen J, Burstein R, Ashina M, Tfelt-Hansen P (2009) Origin of pain in migraine: evidence for peripheral sensitisation. Lancet Neurol 8:679-690.

Parri HR, Crunelli V (2001) Pacemaker calcium oscillations in thalamic astrocytes in situ. Neuroreport 12:3897-3900.

Perrotta A, Serrao M, Sandrini G, Burstein R, Sances G, Rossi P, Bartolo M, Pierelli F, Nappi G (2010) Sensitisation of spinal cord pain processing in medication overuse headache involves supraspinal pain control. Cephalalgia 30:272-284.

Porcaro C, di Lorenzo G, Seri S, Pierelli F, Tecchio F, Coppola G (2017) Impaired brainstem and thalamic high-frequency oscillatory EEG activity in migraine between attacks. Cephalalgia 37:915926.

Rocca MA, Messina R, Colombo B, Falini A, Comi G, Filippi M (2014) Structural brain MRI abnormalities in pediatric patients with migraine. J Neurol 261:350-357.

Russo A, Tessitore A, Giordano A, Corbo D, Marcuccio L, De Stefano M, Salemi F, Conforti R, Esposito F, Tedeschi G (2012) Executive resting-state network connectivity in migraine without aura. Cephalalgia 32:1041-1048 
Salo RA, Miettinen T, Laitinen T, Gröhn O, Sierra A (2017) Diffusion tensor MRI shows progressive changes in the hippocampus and dentate gyrus after status epilepticus in rat - histological validation with Fourier-based analysis. Neurolmage 152:221-236.

Sandrini G, Rossi P, Milanov I, Serrao M, Cecchini AP, Nappi G (2006) Abnormal modulatory influence of diffuse noxious inhibitory controls in migraine and chronic tension-type headache patients. Cephalalgia 26:782-789.

Schwedt TJ, Schlaggar BL, Mar S, Nolan T, Coalson RS, Nardos B, Benzinger T, Larson-Prior LJ (2013) Atypical resting-state functional connectivity of affective pain regions in chronic migraine. Headache 53:737-751.

Sierra A, Laitinen T, Lehtimäki K, Rieppo L, Pitkänen A, Gröhn O (2011) Diffusion tensor MRI with tract-based spatial statistics and histology reveals undiscovered lesioned areas in kainate model of epilepsy in rat. Brain Struct Funct 216:123-135.

Song SK, Sun SW, Ju WK, Lin SJ, Cross AH, Neufeld AH (2003) Diffusion tensor imaging detects and differentiates axon and myelin degeneration in mouse optic nerve after retinal ischemia. Neuroimage 20:1714-1722.
Song SK, Yoshino J, Le TQ, Lin SJ, Sun SW, Cross AH, Armstrong RC (2005) Demyelination increases radial diffusivity in corpus callosum of mouse brain. Neuroimage 26:132-140.

Stankewitz A, Aderjan D, Eippert F, May A (2011) Trigeminal nociceptive transmission in migraineurs predicts migraine attacks. $J$ Neurosci 31:1937-1943.

Teepker M, Kunz M, Peters M, Kundermann B, Schepelmann K, Lautenbacher S (2014) Endogenous pain inhibition during menstrual cycle in migraine. Eur J Pain 18:989-998.

Uggetti C, Squarza S, Longaretti F, Galli A, Di Fiore P, Reganati PF, Campi A, Ardemagni A, Cariati M, Frediani F (2017) Migraine with aura and white matter lesions: an MRI study. Neurol Sci 2017:1113.

Weiller C, May A, Limmroth V, Jüptner M, Kaube H, Schayck RV, Coenen HH, Diener HC (1995) Brain stem activation in spontaneous human migraine attacks. Nat Med 1:658-660.

Zhang J, Wu YL, Su J, Yao Q, Wang M, Li GF, Zhao R, Shi YH, Zhao Y, Zhang Q, Lu H, Xu S, Qin Z, Cui GH, Li J, Liu JR, Du X (2017) Assessment of gray and white matter structural alterations in migraine without aura. J Headache Pain 18:74. 